\title{
Analysis of Rectangular Loop Antenna Printed on Magnetic Plate
}

\author{
Mitsuo Taguchi ${ }^{*(1)}$, Yuki Matsunaga ${ }^{*(2)}$, Shinya Tanaka ${ }^{(1)}$ \\ and Hideaki Shimoda ${ }^{(2)}$ \\ ${ }^{(1)}$ Dept. of Electrical \& Electronic Eng., Nagasaki University \\ (2) Graduate School of Science and Technology, Nagasaki University \\ 1-14 Bunkyo-machi, Nagasaki-shi, 852-8521, Japan \\ ${ }^{*}(2)$ E-mail: d705076k@stcc.nagasaki-u.ac.jp
}

\section{Introduction}

With the rapid development of wireless communication technology such as RFID system, small antennas are desired [1]. By now, many techniques have been proposed to miniaturize antennas, for example, changing the current path, loading the matched circuit, and using dielectric and magnetic materials [2]. Recently, the low loss magnetic materials are developed for the application in the UHF frequencies [3]. Tanaka et al. have analyzed the magnetic material loaded planar inverted $\mathrm{F}$ antenna and showed that the fragmentary magnetic material inserted around the short-pin is mostly effective to miniaturize antenna size [4].

In this paper, a rectangular loop antenna printed on the low loss magnetic plate is numerically and experimentally analyzed. In the numerical analysis, the electromagnetic simulator Micro-Stripes based on the Transmission Line Matrix method is used [5].

\section{Structure of the antenna and analytical conditions}

Figure 1 shows the structure of the analytical model of antenna. The rectangular loop antenna is printed on the surface of the rectangular magnetic plate. The relative permittivity and the relative permeability of the magnetic plate is 14 and 2.4 , respectively. The dielectric loss tangent of it is 0.01 and the magnetic loss tangent is 0.01 at the frequencies from $100 \mathrm{MHz}$ to $1 \mathrm{GHz}$.

In the numerical calculation by Micro-Stripes, the magnetic material is assumed to be isotropic. The calculation conditions are shown in Table 1. Outside of the analytical region, the absorbing boundary is assumed.

In the measurement of input impedance of the antenna, the half of the loop antenna is mounted on the square ground plane of $90 \mathrm{~cm}$ by $90 \mathrm{~cm}$. The antenna is excited by the coaxial feeder behind the ground plane.

\section{Numerical and experimental results}

Figure 2 shows the measured and calculated return loss characteristics of the loop antenna printed on the magnetic plate. The calculated values almost agree with the measured results. 
Figure 3 shows measured return loss characteristics of the rectangular loop antenna with and without the magnetic material. By placing the magnetic plate, the resonant frequency becomes lower from $979 \mathrm{MHz}$ to $767 \mathrm{MHz}$. Figure 4 shows the comparison of calculated return loss characteristics between the loop antenna with and without the magnetic material and with the dielectric material. The relative permittivity and the relative permeability of the dielectric material is 14 and 1, respectively. Figure 5 shows the calculated electric field radiation pattern of loop antenna in free space. Figure 6 shows the calculated electric field radiation pattern of loop antenna printed on magnetic plate. The calculated directivity of loop antenna printed on magnetic plate is $2.1 \mathrm{dBi}$ and that of loop antenna in free space is $2.5 \mathrm{dBi}$.

\section{Conclusion}

The rectangular loop antenna printed on the magnetic plate numerically and experimentally analyzed. The optimization of the geometry of loop antenna and the magnetic plate will be left in the future. The loop antenna printed on the magnetic plate may be suitable for the transponder antenna of RFID system.

$$
\begin{aligned}
& \mathrm{L}_{1}=38 \mathrm{~mm} \\
& \mathrm{~L}_{2}=132 \mathrm{~mm} \\
& \mathrm{~L}_{3}=28 \mathrm{~mm} \\
& \mathrm{~L}_{4}=122 \mathrm{~mm} \\
& \mathrm{~W}=2 \mathrm{~mm} \\
& \mathrm{t}=1.3 \mathrm{~mm}
\end{aligned}
$$

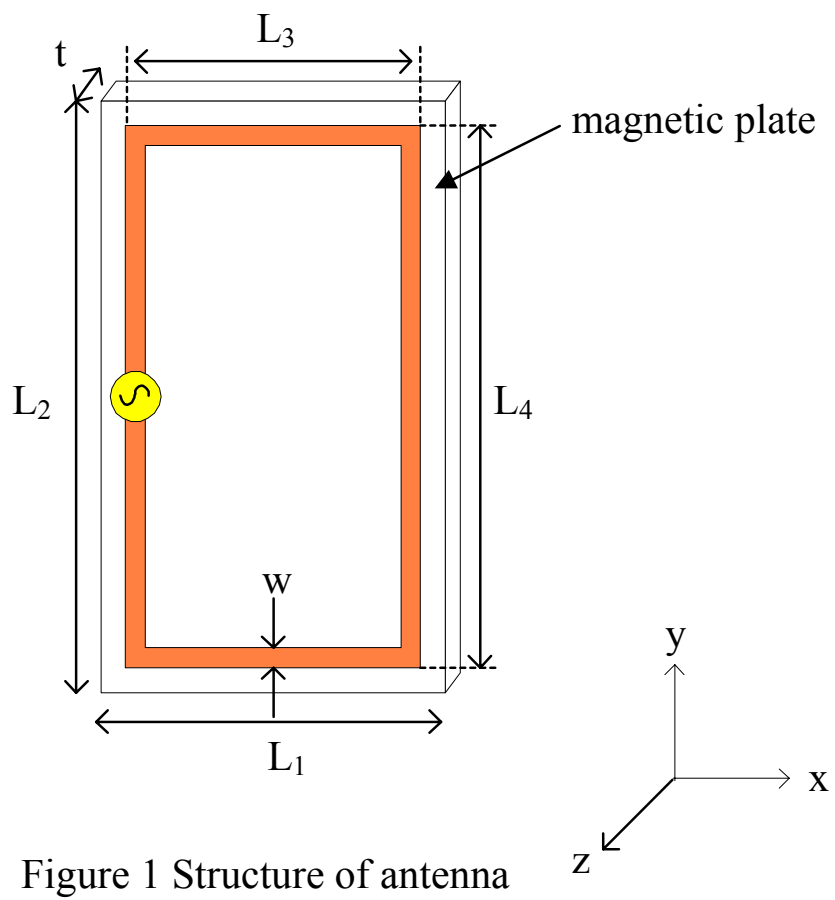

Table 1 Analytical condition

\begin{tabular}{|c|c|c|c|}
\hline & Analytical Region & Maximum Cell Size & Minimum Cell Size \\
\hline $\mathrm{x}$-axis & 330 & 18 & 0.1 \\
\hline $\mathrm{y}$-axis & 420 & 17.8 & 0.04 \\
\hline z-axis & 300 & 18 & 0.1 \\
\hline
\end{tabular}




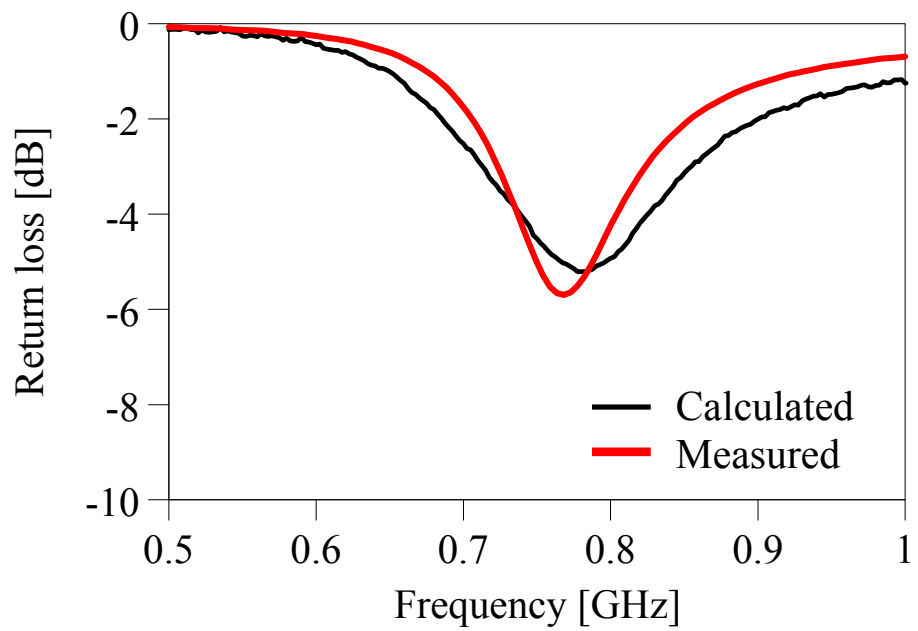

Figure 2 Return loss characteristics of loop antenna printed on magnetic plate.

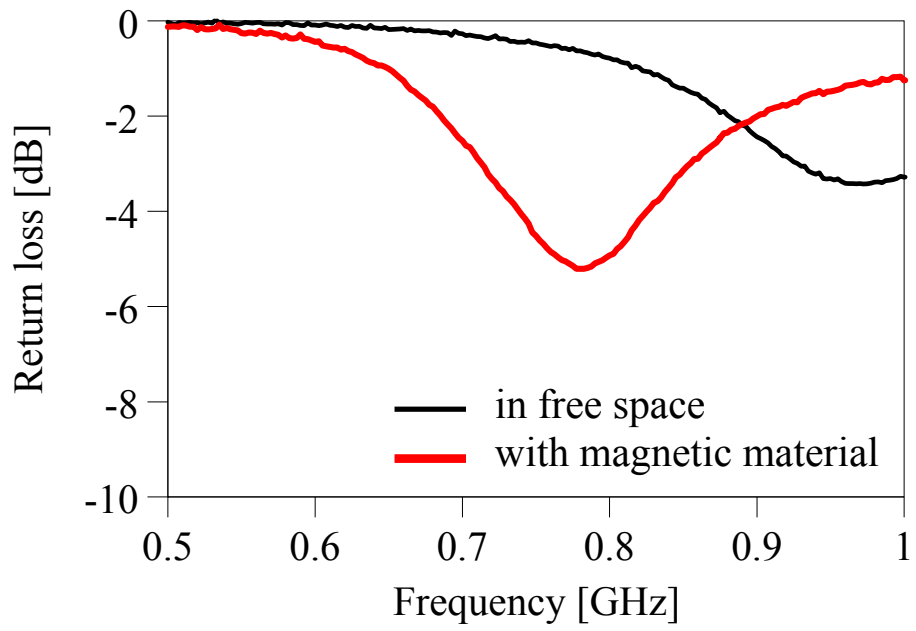

Figure 3 Measured return loss characteristics.

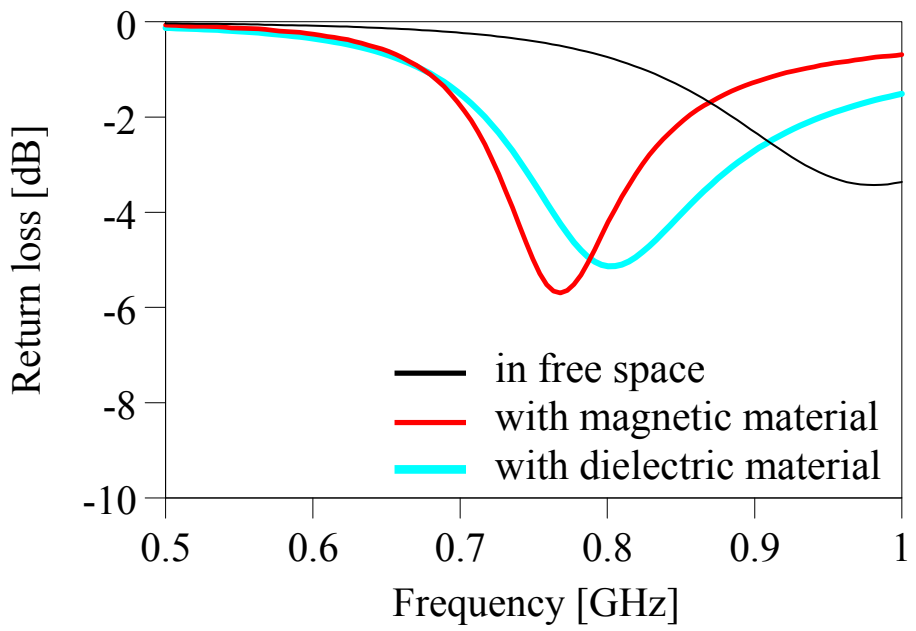

Figure 4 Calculated return loss characteristics. 


\section{References}

[1] K. Finkenzeller: "RFID handbook", John Wiley \& Sons, 2003.

[2] H. Arai: "Small antennas: Downsizing techniques and its index factor", IEICE Trans. on Communications, vol. J87-B, no. 9, pp. 1140-1148, Sept. 2004.

[3] N.Kitahara, T.Mizumoto: "Magnetic ceramics materials with high permittivity and low loss", IEICE Trans. on Communications, vol.J86-C, no4, pp.450-456, April 2003.

[4] T. Tanaka, S. Hayashida, K. Imamura, H. Morishita, and Y. Koyanagi: "A study of miniaturization of antenna for handset utilizing magnetic materials", IEICE Trans. on Communications, vol. J87-B, no. 9, pp. 1327-1335, Sept. 2004.

[5] "Micro-Stripes Reference Manual Release 6.0", Flomerics Limited, 2002

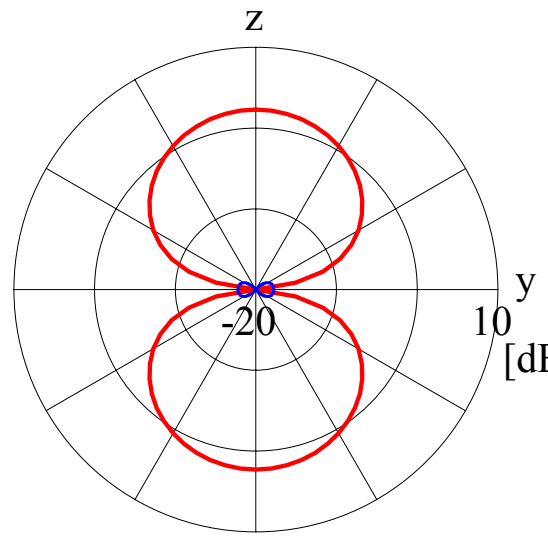

(a) yz-plane

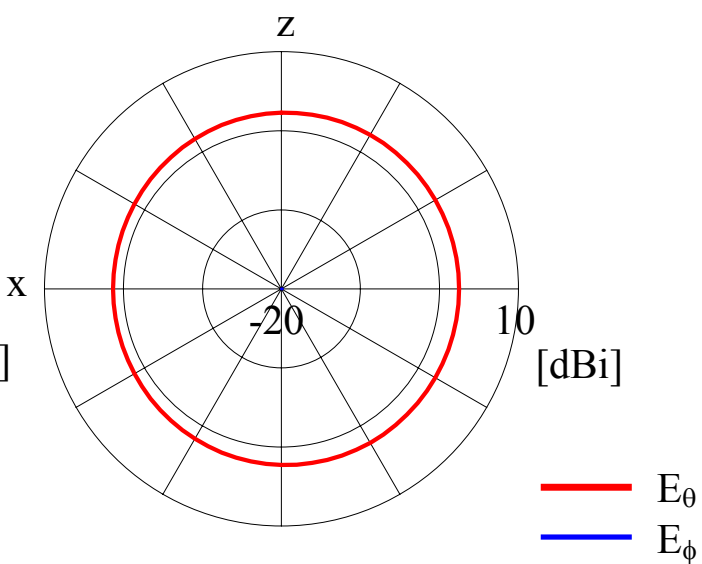

(b) xz-plane

Figure 5 Electric field radiation pattern of loopantenna in free space.

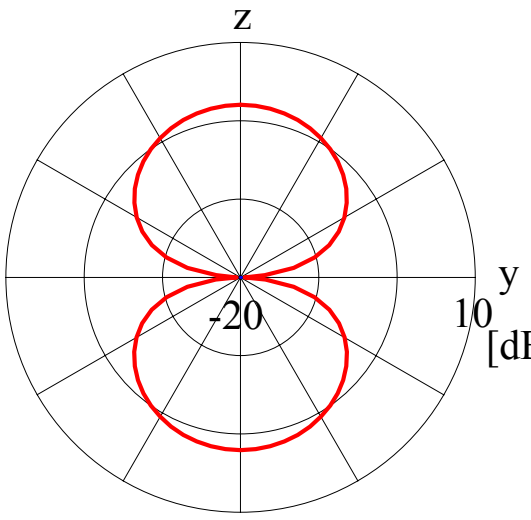

(a) yz-plane

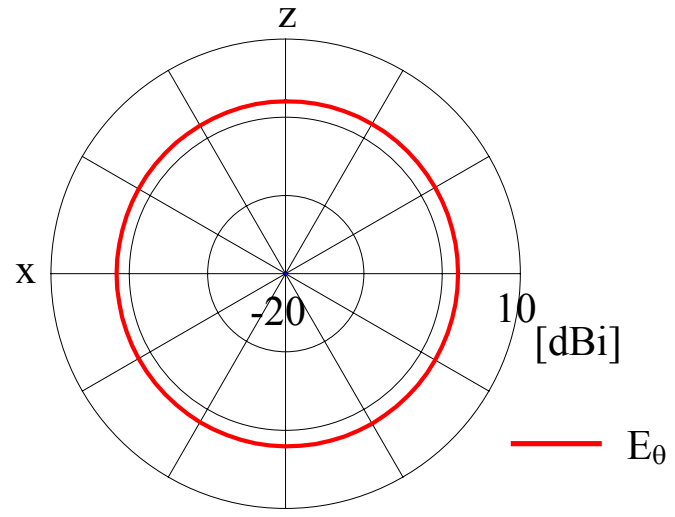

(b) xz-plane

Figure 6 Electric field radiation pattern of loop antenna printed on magnetic plate. 